RISING CO2 Will forests need vitamins? Commun. Biol. 3, 125 (2020)

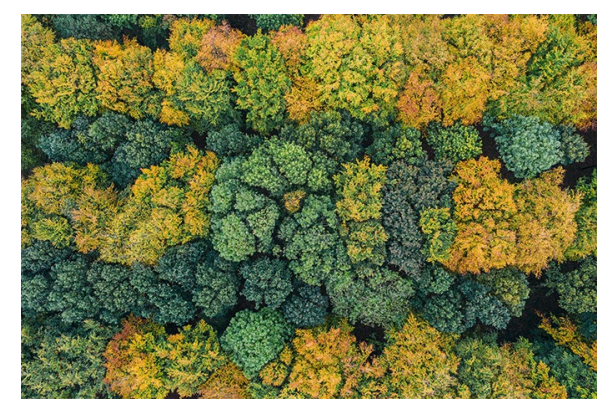

Credit: Justin Paget / DigitalVision / Getty

Atmospheric $\mathrm{CO}_{2}$ has increased from 350 to $410 \mathrm{ppm}$ in the last three decades, which has been associated with greater plant growth and more efficient photosynthesis. The impacts of elevated $\mathrm{CO}_{2}$ on vegetation are, however, not all positive. Enhanced growth and photosynthesis can dilute nutrient concentrations in leaves, and plant physiological responses to elevated $\mathrm{CO}_{2}$ and associated climate changes can limit nutrient uptake. Together with other global change factors, rising $\mathrm{CO}_{2}$ may thus negatively impact the nutritional quality of forests.

To determine this effect, Josep Penuelas from CREAF-CSIC-UAB, Spain, and colleagues measured changes in leaf elemental composition over the past 30 years for European trees and investigated underlying drivers of these changes. They find the nutrient quality of leaves has generally decreased throughout Europe, with the greatest decreases observed in Mediterranean and temperate forests due to rising $\mathrm{CO}_{2}$. In contrast, concentrations of nitrogen, sulfur and calcium have increased in boreal forests because temperature and precipitation have become more favourable. These results indicate a potential negative feedback on forest carbon sequestration under climate change, particularly for southern European forests.

https://doi.org/10.1038/s41558-020-0777-1

\section{PRO-ENVIRONMENTAL BEHAVIOUR}

Competing models

\section{J. Environ. Psychol. 68, 101408 (2020)}

Two behaviour change theories have been used to understand the determinants of pro-environmental behaviour. The theory of planned behaviour (TPB) posits that individuals decide to engage in pro-environmental behaviour based on attitudes, subjective norms and perceived behavioural control; the valuebelief-norm (VBN) theory suggests that pro-environmental behaviours are a class of prosocial behaviours driven by perceptions of moral obligation to behave a certain way, and the potential negative consequences for others of neglecting to do so. Although both models have been empirically validated in the context of adaptation and mitigation behaviour, their relative predictive power has not been tested.

Lu Zhang from Huazhong Agricultural University, China, and colleagues surveyed rice farmers in China on their intended

CONSERVATION PRIORITIES Climate or biodiversity? Phil. Trans. R. Soc. B 375, 20190128 (2020)

Climate change and the rapid rate of biodiversity loss are two defining environmental crises of our time, but approaches to deal with one often neglect the other. In particular, climate change mitigation measures often focus on protecting high-carbon ecosystems, irrespective of their biodiversity value. As such, global biodiversity and climate objectives may be in poor agreement, or even in conflict.

In order to integrate these two objectives, Carolina Soto-Navarro and colleagues identified global areas of potential co-benefits for climate change mitigation and biodiversity conservation. Importantly, they measured the multiple dimensions of biodiversity, including both species- and ecosystem-based values. To do so, they first developed a high-resolution global map of above- and below-ground carbon storage. This was subsequently integrated with biodiversity values representing either proactive or reactive conservation approaches. The authors identify 'hotspots', where areas of highest value for carbon storage and biodiversity values overlap, as well as highlighting the risk to these hotspots that remain largely unprotected. It is hoped that this work will facilitate implementation of integrated conservation targets.

https://doi.org/10.1038/s41558-020-0780-6 agricultural adaptation and mitigation behaviours as well as socio-psychological variables. Structural equation modelling showed that the TPB was a better predictor of climate change adaptation, whereas the VBM theory was a better predictor of climate change mitigation. These results suggest that while both adaptation and mitigation are effective responses to climate change, decisions to engage in such behaviours are driven by different psychological mechanisms.

https://doi.org/10.1038/s41558-020-0779-Z

\section{GLACIAL LAKES}

\section{Hazards in the Himalayas \\ Water Resour. Res. 56, e2019WR026533 (2020)}

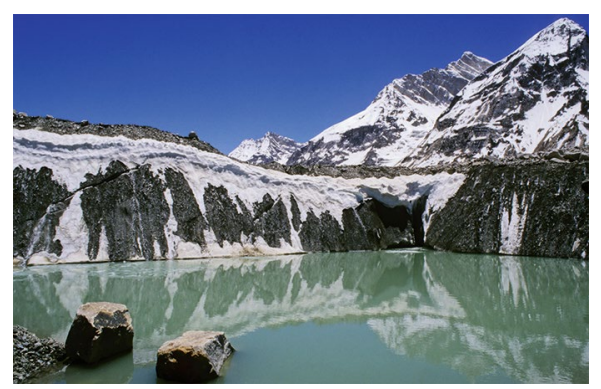

Credit: Dinodia Photos / Alamy Stock Photo

Recent warming has caused glacier retreat in high-mountain Asia, with a corresponding increase in the size and number of glacial lakes dammed by glaciers or their debris. A glacial lake outburst flood (GLOF) occurs when these dams fail - for example, from melting, avalanches or upstream GLOFs. These events can have major downstream flooding impacts, and it is important to identify lakes at highest risk.

Saket Dubey and Manish Kumar Goyal of the Indian Institute of Technology Indore, India, use satellite data with elevation and flood models to gauge the hazard risk of 329 glacial lakes in the Indian Himalayas. The authors report a nearly $16 \%$ increase in lake area during 1993-2018, driven by one-fifth of identified lakes. They estimate around $22 \%$ of these lakes present a high or very high hazard risk due to both GLOF event likelihood and downstream impacts. An additional 59\% show moderate risk. Such findings can help identify which lakes need further risk analysis and assist in regional climate change adaptation strategies.

https://doi.org/10.1038/s41558-020-0778-0

Tegan Armarego-Marriott, Alyssa Findlay, Baird Langenbrunner and Jenn Richler 